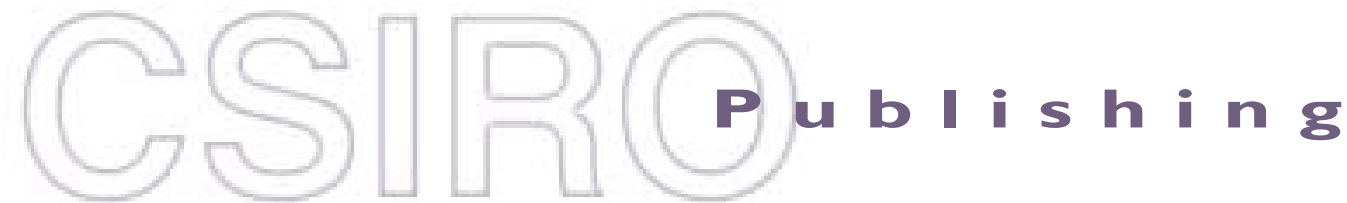

\section{Publications of the Astronomical Society of Australia}

Volume 19, 2002

(C) Astronomical Society of Australia 2002

An international journal of astronomy and astrophysics

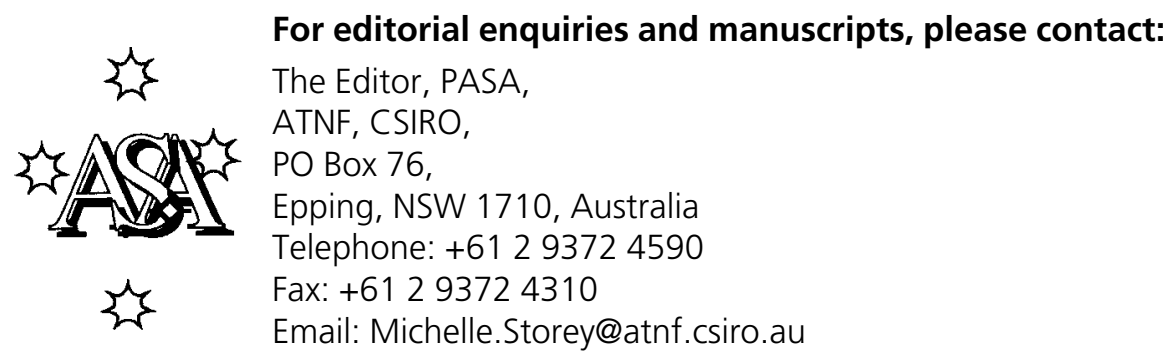

For general enquiries and subscriptions, please contact: CSIRO Publishing PO Box 1139 (150 Oxford St)

Collingwood, Vic. 3066, Australia

Telephone: +6139662 7666

Fax: +61 396627555

Email: publishing.pasa@csiro.au

C S I RO

PUBLISHING Published by CSIRO Publishing

for the Astronomical Society of Australia

www.publish.csiro.au/journals/pasa 


\title{
X-ray Variability of Blazars
}

\author{
Elena Pian \\ INAF, Astronomical Observatory of Trieste, Via G. B. Tiepolo 11, I-34131 Trieste, Italy \\ pian@ts.astro.it
}

Received 2001 July 31, accepted 2001 November 8

\begin{abstract}
Critical progress in our understanding of high energy emission from AGN has been determined in the last 10 years by X-ray monitoring campaigns with many space missions, notably ROSAT, ASCA, RXTE, BeppoSAX, and XMM, often in conjunction with observations at other frequencies. The emphasis of the present review is on recent findings about X-ray variability of blazars. Among AGN, these exhibit the largest amplitude variations of the X-ray emission, often well correlated with variations at higher energies ( $\mathrm{GeV}$ and $\mathrm{TeV}$ radiation). The accurate sampling of the $\mathrm{X}$-ray spectra over more than three decades in energy, made possible by the wide energy range of BeppoSAX, has also shown strong spectral variability in blazar active states, suggesting extreme electron energies and leading to the identification of a class of 'extreme synchrotron' sources.
\end{abstract}

Keywords: radiation mechanisms: non-thermal $-\mathrm{X}$-rays: galaxies

\section{Introduction}

The multiwavelength continuum emission of blazar type AGN is dominated by non-thermal radiation in a relativistic jet pointing close to the line of sight. Magnification of the light intensity and variation amplitude, and foreshortening of intrinsic variability time scales, due to aberration effects, make blazars the most luminous and variable AGN at all frequencies and the most direct probe of galactic nuclear continuum emission. The amplitude of flux density variations increases as a function of frequency. Sensitive X-ray observations of bright blazars are therefore effective in measuring temporal and spectral properties on time scales as short as hours or even minutes. X-ray variability on the shortest time scales allows us to derive the sizes of the inner nuclear regions and to trace the kinematics of the jet, and thereby study how the relativistic particles are accelerated and injected in the emission region and how efficiently they cool thereafter and escape. Combined with simultaneous observations at other wavelengths, X-ray light curves carry information on multiwavelength spectral variability and on temporal delays of emission at different frequencies. These can be used to test the models for the production of the blazar continuum.

The spectral energy distributions (SEDs) of blazars are typically double-peaked (in a $v f_{v}$ representation), with a first component attributed to synchrotron radiation, the maximum of which is located at IR to soft X-ray frequencies, and a second component correspondingly peaking at $\mathrm{GeV}$ to $\mathrm{TeV}$ energies, produced through inverse Compton scattering (Figure 1, left). According to whether the peak of their first component is at IR or UV/soft X-ray wavelengths, the sources have been defined as Low-Energy or High-Energy Peaked Blazars (Padovani \& Giommi 1995), or as 'red' or 'blue' blazars (Fossati et al. 1998). However, rather than exhibiting a neat distinction between red and blue sources, the blazar population presents a continuity of properties between these two extrema, all correlating with the bolometric luminosity.

High energy peaked blazars have lower bolometric luminosities and smaller ratios between high energy and low energy components (i.e. smaller inverse Compton dominance). Ghisellini et al. (1998) identify the ambient (broad emission line region or accretion disk) photon density as the parameter governing these correlations: in sources with lower ambient photon densities (blue blazars) the high energy electrons cool via scattering off synchrotron photons (synchrotron self-Compton mechanism, SSC), while for high ambient photon densities the inverse Compton scattering off external radiation prevails (red blazars).

In blue blazars the radiation efficiency is smaller than in red ones, and therefore in the former sources electrons can attain higher energies, thus synchrotron spectra peak at higher frequencies, and the overall luminosity is lower than in red blazars (however, Urry 1999 discusses some caveats against this scenario). The difference in synchrotron luminosities may be determined by different degrees of relativistic beaming. Boettcher \& Dermer (2002) interpret the increasing spectral hardness with decreasing luminosity of blazars in an evolutionary scenario of increasing reduction of the black hole accretion power with time (see also Maraschi 2001).

In the X-ray spectral range the two contributions from synchrotron and inverse Compton components overlap, with the former dominating in blue blazars, and the latter in red blazars (see Donato et al. 2001 for a recent comprehensive review of blazar X-ray spectra). Therefore, in blue blazars photons up to $10 \mathrm{keV}$, and occasionally $100 \mathrm{keV}$ or more, especially in outburst, represent the highest energy tail of the synchrotron radiation. Being produced by the most energetic electrons, they are most sensitive to variations in the acceleration and cooling processes, and are thus expected to exhibit the most rapid 

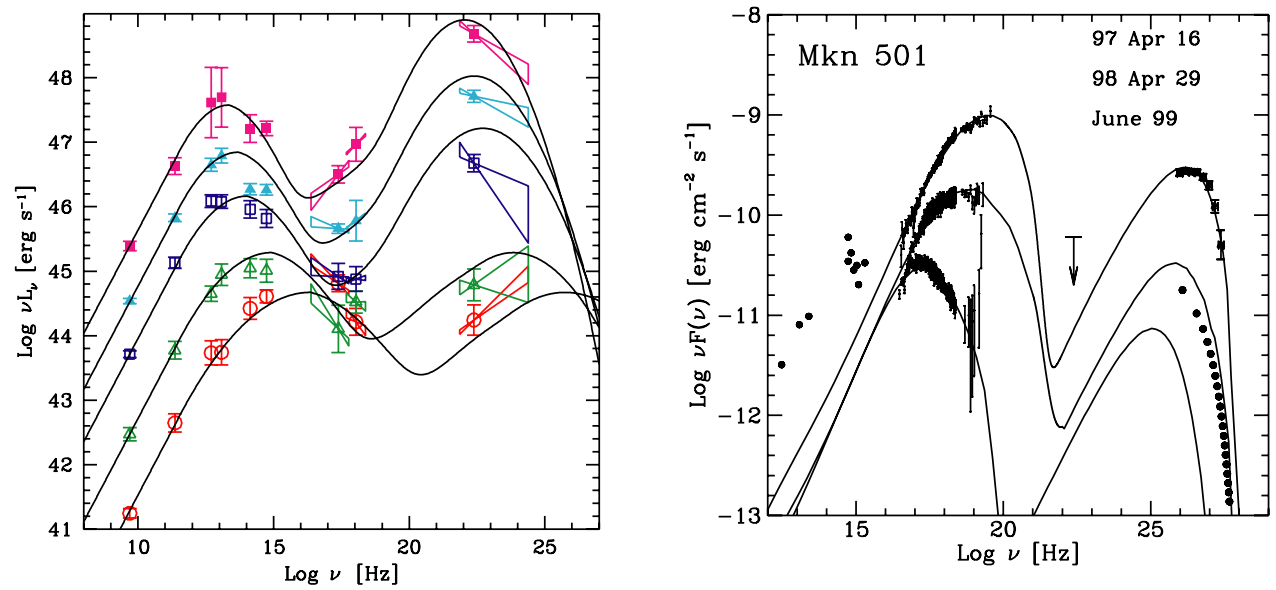

Figure 1 Left: Average SEDs of blazars binned according to radio luminosity (Fossati et al. 1998). The curves represent the parameterisation proposed by Donato et al. (2001). Right: SEDs of Mkn 501. The solid curves are spectra calculated with the homogeneous SSC model (see Tavecchio et al. 2001 for details on the data and on the model parameters). The filled circles at TeV energies represent the HEGRA 1998-1999 data (Aharonian et al. 2001). The assumed emitting region is homogeneous and the general aim of the modelling is to reproduce the observed variability with a minimum number of physical parameter changes. These approximations may not be totally rigorous and valid (i.e. the emitting region could have a frequency dependent size, with lower frequency radiation being produced in larger regions). This may account for the departure of the model from data at optical and longer wavelengths. Moreover, these may be contaminated by the host galaxy emission (from Tavecchio et al. 2001).

and largest amplitude variability. In a few of these blazars the inverse Compton component, produced through SSC, is detected up to $\mathrm{TeV}$ energies.

In red blazars, the X-ray spectrum is still due to SSC, while external Compton dominates at the higher frequencies (Takahashi, Madejski, \& Kubo 1999). In the last 10 years, the blazar monitoring by X-ray satellites ROSAT, ASCA, RXTE, BeppoSAX, and recently $\mathrm{XMM}$, has produced many results on variability, as reviewed, e.g. by Ulrich, Maraschi, \& Urry (1997), Urry (1999), Sambruna (1999), McHardy (1999), Maraschi et al. (2000), and Maraschi \& Tavecchio (2001). The best studied red blazars present X-ray variability correlated with optical and gamma rays, although the variation amplitude is usually smaller than in gamma-rays (e.g., Wehrle et al. 1998; Ghisellini et al. 1999). The hard X-ray spectral variability is modest (Sambruna et al. 1997; Lawson et al. 1999; Hartman et al. 2001; Ballo et al. 2002). For some objects (intermediate blazars) the X-ray observations have simultaneously sampled the synchrotron and the SSC components at soft and hard X-rays, respectively (Giommi et al. 1999; Tagliaferri et al. 2000; Ravasio et al. 2002; Tanihata et al. 2000).

Blue blazars present a higher amplitude $\mathrm{X}$-ray emission variability than red blazars (factors of up to 30 with respect to quiescence), accompanied by different spectral variability (compare the remarkable changes of spectral slope and synchrotron peak frequency in Mkn 501, Pian et al. 1998, with the modest peak frequency variations in PKS 2005489, Perlman et al. 1999; Tagliaferri et al. 2001). In the present paper I will summarise some of the most recent results obtained for the brightest and best monitored BL Lacs Mkn 501, Mkn 421, and PKS 2155-304, all of which belong to the class of blue blazars.

\section{Mkn 501}

This source has been observed by BeppoSAX in April 1997, April-May 1998, and June 1999. On the first occasion it exhibited an outburst accompanied by dramatic spectral hardening: the synchrotron peak energy was observed to reach $100 \mathrm{keV}$ or more, i.e. almost three orders of magnitude larger than in its quiescent state (Catanese et al. 1997; Pian et al. 1998). The following year, the peak energy was found at $20 \mathrm{keV}$ and the emission intensity above $1 \mathrm{keV}$ was lower. The fact that, despite the radiation losses, the synchrotron peak was still at such high energies one year after the outburst indicates the presence of efficient, continuously active mechanisms of particle acceleration in this source.

In June 1999 BeppoSAX recorded an 'inactive' state, i.e. similar to the historical one, both in emission level and synchrotron peak energy $(\sim 0.5 \mathrm{keV})$, and characterised by modest (amplitude of 10-20\%) intraday flux variability (Tavecchio et al. 2001). In Figure 1, right, the X-ray spectra are shown together with the simultaneous TeV observations. The X-ray spectra are best described by curved and continuously steepening laws. Their variability can be reproduced by changes of the electron break energy only (i.e. the energy of the electrons which emit at the spectral maximum). As frequently noted in blazars (see also Section 3), the X-ray spectral index and peak energy correlate well with the source intensity, flatter index and higher peak energy corresponding to brighter states.

The X-ray spectral index and peak energy correlate very well with the source intensity, such that a flatter index and higher energy correspond to higher intensity.

The good correlation between intensity and spectral index holds also when previous GINGA and ASCA observations are considered together with BeppoSAX data 
(Kataoka et al. 1999). The X-ray and TeV intensities correlate well with no measurable time lag larger than one day. This suggests that the same electron population is responsible for the $\mathrm{X}$ - and gamma-ray emission through the SSC mechanism in a homogeneous region (Tavecchio, Maraschi, \& Ghisellini 1998; Tavecchio et al. 2001; Petry et al. 2000). The ensuing prediction for the correlated behaviour of X-ray and $\mathrm{TeV}$ flux densities in different states (Tavecchio et al. 2001) is in good agreement with the simultaneous RXTE and TeV HEGRA observations in 1997 (see Figure 6 in Krawczynski et al. 2000).

Strong correlation between spectral hardness and intensity has been observed by RXTE as well in June 1998, when a rapid shift in synchrotron peak energy was also detected during a brightening. The simultaneous X-ray and $\mathrm{TeV}$ flux and spectral variability points, again, to the SSC origin of the multiwavelength emission (Sambruna et al. 2000).

In July 1997, when Mkn 501 was close to its historically largest X-ray intensity, RXTE observations of the source revealed unusual anti-correlation between spectral hardness and intensity (i.e. flatter spectrum for dimmer intensity). At that epoch the source exhibited variations of up to $30 \%$ over a few hours (Lamer \& Wagner 1998). Remarkably rapid variability has been detected during the May 1998 RXTE campaign, when the blazar flared by factors 1.6 and 2 in $\sim 200$ seconds in the $2-10 \mathrm{keV}$ range and 10-15 keV, respectively (Catanese \& Sambruna
2000). With the event is associated significant spectral variability.

\section{Mkn 421}

A 1 day flare was detected in this source simultaneously in X-rays by BeppoSAX and at $\mathrm{TeV}$ energies by the Whipple Cerenkov telescope in April 1998 at the beginning of a 10 day multiwavelength monitoring campaign, involving also the ASCA, EUVE, and RXTE satellites (Takahashi et al. 2000). The X-ray and TeV light curves are well correlated with no temporal lag larger than 1.5 hours (Figure 2, left), indicating that the radiation at the two frequencies is produced by the same electron population via SSC (Maraschi et al. 1999). The observed difference in the flare decay times in X-rays and $\mathrm{TeV}$ may be due to light travel time effects adding to radiative time scales (Chiaberge \& Ghisellini 1999), or to the inhomogeneity of the emitting region (see also the simultaneous RXTE and TeV observations of June 2000 by Krawczynski et al. 2001).

Within the X-ray band the flare decay is achromatic, if a stationary underlying emission component is postulated (Fossati et al. 2000a). The temporally resolved spectra during the X-ray flare allowed Fossati et al. (2000b) to fully map, for the first time in any blazar, the increase of the synchrotron peak energy during the rising part of the flare and its subsequent decrease during the decay (Figure 2, right).
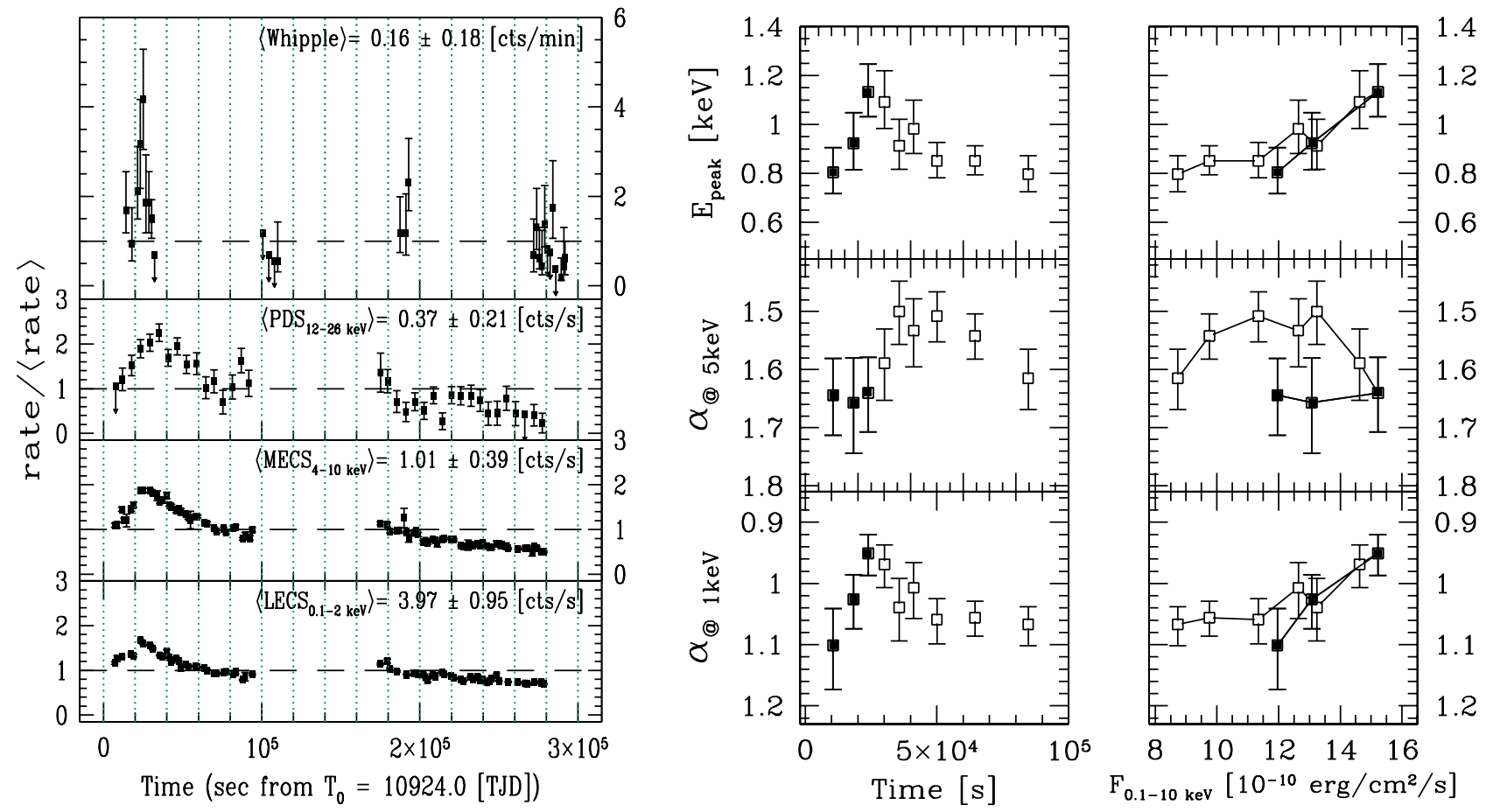

Figure 2 April 1998 flare of Mkn 421. Left: light curves at X-ray (BeppoSAX) and TeV (Whipple) energies on 21-24 April 1998. All count rates are normalised to their respective averages (from Maraschi et al. 1999). Right, top to bottom: the energy of the peak of the synchrotron component, and the photon spectral indices at 5 and at $1 \mathrm{keV}$ versus time (left column) and de-absorbed 0.1-10 keV intensity (right column). The solid black symbols represent the behaviour before the top of the flare, while empty dots represent the spectral evolution during the decay. The data points are connected to show the time sequence. A shift of the synchrotron peak and a delay in the response of the spectrum at $5 \mathrm{keV}$ can be clearly seen (from Fossati et al. 2000b). 
Unlike in Mkn 501, where the synchrotron peak during the outburst can reach $100 \mathrm{keV}$ or more, in Mkn 421 the maximum energy reached by the synchrotron peak is $1 \mathrm{keV}$. An indication that it may have shifted up to $10 \mathrm{keV}$ or beyond is present in May 2000 BeppoSAX data, when the source was also detected in its historical maximum X-ray brightness $\left(F_{2-10 \mathrm{keV}}=1.2 \times 10^{-9} \mathrm{erg} \mathrm{s}^{-1} \mathrm{~cm}^{-2}\right.$, Fossati 2001). It is interesting to note that Mkn 421 presents larger amplitude short term variability than Mkn 501, which suggests that maximum synchrotron peak energy and short time scale variability may have complementary roles in dissipating the jet energy.

It is generally observed that the X-ray intensity and spectral index variations in blazars are correlated, in the sense of spectral hardening during brightening. It has been noted for some time (see e.g. Sembay et al. 1993) that the changes describe different paths in the index-intensity plane, according to whether they refer to the increasing or decreasing portion of a flare. This is seen also during the April 1998 flare of Mkn 421, which exhibits an 'anticlockwise' pattern of intensity vs spectral index (middle and bottom panels of the right side of Figure 2, right). For Mkn 421 this is less common than the opposite 'clockwise' pattern (Takahashi et al. 1996; Malizia et al. 2000).

The emission of soft X-rays $(\lesssim 2 \mathrm{keV})$ is generally well correlated with and lags that of hard X-rays ( $\gtrsim 4 \mathrm{keV})$ by a variable interval (from 3-4 ks down to $\sim 300 \mathrm{~s}$ ) as determined with cross-correlation methods (Malizia et al. 2000; Takahashi et al. 2000; Brinkmann et al. 2001). The soft lag is interpreted as a consequence of the synchrotron cooling, which is more rapid at higher energies. The reversal of this temporal correlation has been observed in 1997 and April 1998 (Treves et al. 1999; Fossati et al. 2000a), suggesting that hard lags may be associated with anti-clockwise intensity spectrum patterns, while soft lags are associated with clockwise patterns. Hard lags may originate when the acceleration time scale approaches the cooling time scale, so that electrons appear first at low energies and gradually build up at higher energies (Kirk, Rieger, \& Mastichiadis 1998; Takahashi et al. 2000).

We note, however, that Edelson et al. (2001) have put into question temporal lags of the order of $\sim 1$ hour, when obtained by applying cross-correlation methods to light curves measured by satellites with low Earth orbits (like ASCA and BeppoSAX).

Mkn 421 flares daily, with a characteristic time scale of 12 hours, as determined from the structure function analysis of the ASCA data in April 1998 (Takahashi et al. 2000; a similar result has been obtained with previous ASCA observations in 1994, Takahashi et al. 1996, and with XMM observations in May 2000, Brinkmann et al. 2001). However, $50 \%$ variations of 4-5 hours time scale are typical (Guainazzi et al. 1999; Fossati et al. 2000a; Krawczynski et al. 2001). The symmetric shape of the X-ray flares detected by BeppoSAX and ASCA imply that the cooling time scales are shorter than the light crossing time through the emitting region, so that the latter dominates the variability.

\section{PKS 2155-304}

A blue, relatively nearby $(z \simeq 0.1)$ blazar, and one of the brightest at UV and X-ray frequencies, PKS 2155-304 was expected to be a $\mathrm{TeV}$ emitter and has indeed been detected at these energies in September-November 1996 by the Cerenkov telescope Mark 6 (Narrabri, Australia) and, with a larger intensity, simultaneously with EGRET and BeppoSAX in November 1997 (Chadwick et al. 1999; Chiappetti et al. 1999; Vestrand \& Sreekumar 1999). The $\mathrm{X}$-ray state observed by BeppoSAX is one of the highest ever detected, though lower than that observed by RXTE before the start of the BeppoSAX campaign. A pure SSC model in a homogeneous region accounts well for the X-ray and higher energy radiation, with variability produced solely by changes in electron break energy (Figure 3).

A comparative temporal analysis on the X-ray light curves of PKS 2155-304 in 1994 (ASCA, Kataoka et al. 2000), 1996, and 1997 (BeppoSAX, Giommi et al. 1998; Chiappetti et al. 1999) has been accomplished by Zhang et al. (1999), who found power density spectra of slope similar to those of Seyfert galaxies $\left(f^{-1.7}\right.$, e.g. Edelson \& Nandra 1999). The minimum variability time scale determined by the power density spectrum analysis in the $1.5-10 \mathrm{keV}$ range is $\sim 1000 \mathrm{~s}$, consistent with the minimum measured doubling time of the flux.

The degree of variability is found to be a function of brightness, with shorter doubling times and larger fractional variability parameter for brighter states. Similarly, the temporal delays between soft and hard X-rays, determined with different correlation methods, are shorter

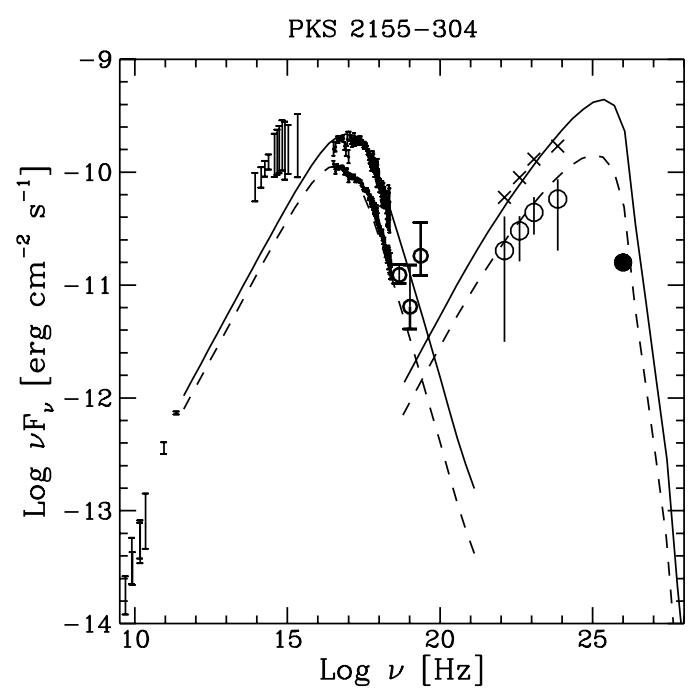

Figure 3 SEDs of PKS 2155-304. The BeppoSAX spectra in the high and low states and the TeV point refer to simultaneous observations in November 1997. The vertical bars encompass the range between the minimum and maximum value in a compilation of radio, optical, and UV data. The X-ray spectra are fitted with a homogeneous SSC model (solid and dashed curves, respectively). A comment similar to that made for Mkn 501 (Figure 1, right) applies here to the inconsistency of the model with UV/optical data (from Chiappetti et al. 1999). 
for higher source intensity (see, however, Section 3 and Edelson et al. 2001).

Both positive and negative temporal lags between soft and hard X-rays have been measured by RXTE for PKS 2155-304 during successive flares in May 1996, and correspondingly a clockwise or anti-clockwise loop, respectively, is seen in the intensity vs hardness ratio plane (Sambruna 1999). This, as in the case of Mkn 421, can be interpreted in terms of a different duration of the acceleration process with respect to the electron cooling (Kirk et al. 1998).

The variable temporal lags between soft and hard $\mathrm{X}$-ray emission may be related to a different geometry of the emitting region in different states of the source (Zhang et al. 1999), requiring a time-dependent SSC model to reproduce the SED (Kataoka et al. 2000). Alternatively, Georganopoulos \& Marscher (1998) have proposed that the different temporal lags observed in 1991 and 1994 between light curves of PKS 2155-304 at UV and X-ray frequencies (Edelson et al. 1995; Urry et al. 1997) can be reproduced by flaring components superimposed on a similar steady state of the jet, and produced by different energy distributions of freshly injected electrons.

It is striking that in this source, similar patterns of X-ray variability are seen, spaced apart by many years (Brinkmann et al. 2000), suggesting recurrent conditions in the mechanism of jet powering and activity development. This also weakens the microlensing hypothesis as the origin of intraday variability in this source (Urry et al. 1993).

\section{Conclusions}

The X-ray campaigns, coordinated with $\mathrm{TeV}$ telescopes, have determined a crucial progress toward our understanding of blazars and have shown the importance of $\mathrm{X}$-ray instruments with wide spectral range and good temporal resolution. The correlated variability at X-ray and $\mathrm{TeV}$ energies can be reproduced by the changes of a single parameter, namely the break energy of the relativistic electron distribution. In some sources, especially (but not necessarily) during outburst, this parameter can attain extremely high values $\left(\gamma_{b}>10^{6}\right)$, resulting in synchrotron peaks beyond $1 \mathrm{keV}$ (Mkn 421), and even up to 50-100 keV, as in Mkn 501, 1ES 2344+514 (Giommi, Padovani, \& Perlman 2000), and 1ES 1426+428 (Costamante et al. 2001).

More detections of these 'extreme synchrotron blazars' are expected with the advent of the INTEGRAL satellite for soft gamma-ray astronomy. These sources are expected to be $\mathrm{TeV}$ emitters, and therefore should be suitable targets for the next generation of sensitive Cerenkov telescopes. Simultaneous observations at X-ray and $\mathrm{TeV}$ energies should allow us to measure the correlation of the light curves on shorter time scales than it has been possible so far and identify the relation between source intensity and variable temporal lags. Similar coordinated observations of red blazars, which are not expected to be strong
$\mathrm{TeV}$ emitters, will receive renewed interest after the launch in the near future of satellites sensitive in the $\mathrm{MeV}-\mathrm{GeV}$ range, i.e. AGILE and GLAST.

Finally, longer and denser monitoring of blazars will allow a better test of the hadronic models of blazar emission. These predict gamma rays to be due to proton synchrotron radiation (Aharonian 2000), or to pion production by accelerated protons interacting with turbulent fluctuations in the magnetic field (Mannheim 1998), or with ambient radiation fields (Donea \& Protheroe 2002). In hadronic models, correlated variability at X-ray and $\mathrm{TeV}$ energies may arise from less restrictive sets of parameters than in leptonic models (SSC, or synchrotron and external Compton). However, hadronic and leptonic models are clearly separated in the parameter space, so that it should be possible to distinguish them via a modelindependent estimate of the magnetic field and plasma Doppler factor (Rachen 2000).

\section{Acknowledgments}

I thank A. Celotti, L. Chiappetti, G. Fossati, G. Ghisellini, F. Tavecchio, and A. Treves for valuable input, L. Maraschi for a careful reading of the manuscript, and the workshop organisers for a pleasant, stimulating, and constructive meeting.

\section{References}

Aharonian, F. 2000, NewA, 5, 377

Aharonian, F., et al. 2001, ApJ, 546, 898

Ballo, L., et al. 2002, ApJ, 567, in press (astro-ph/0111499)

Boettcher, M., \& Dermer, C. D. 2002, ApJ, 564, 86

Brinkmann, W., Gliozzi, M., Urry, C. M., Maraschi, L., \& Sambruna, R. 2000, A\&A, 362, 105

Brinkmann, W., et al. 2001, A\&A, 365, L162

Catanese, M., et al. 1997, ApJ, 487, L143

Catanese, M., \& Sambruna, R. M. 2000, ApJ, 534, L39

Chadwick, P. M., et al. 1999, ApJ, 513, 161

Chiaberge, M., \& Ghisellini, G. 1999, MNRAS, 306, 551

Chiappetti, L., et al. 1999, ApJ, 521, 552

Costamante, L., et al. 2001, A\&A, 371, 512

Donato, D., Ghisellini, G., Tagliaferri, G., \& Fossati, G. 2001, A\&A, 375,739

Donea, A.-C., \& Protheroe, R. J. 2002, PASA, 19, 39

Edelson, R. A., et al. 1995, ApJ, 438, 120

Edelson, R. A., \& Nandra, K. 1999, ApJ, 514, 682

Edelson, R. A., Griffiths, G., Markowitz, A., Sembay, S., Turner, M. J. L., \& Warwick, R. 2001, ApJ, 554, 274

Fossati, G., Maraschi, L., Celotti, A., Comastri, A., \& Ghisellini, G 1998, MNRAS, 299, 433

Fossati, G., et al. 2000a, ApJ, 541, 153

Fossati, G., et al. 2000b, ApJ, 541, 166

Fossati, G. 2001, Proc. of "X-ray Astronomy 2000" (Palermo, September 2000), Eds. R. Giacconi, L. Stella, and S. Serio, ASP Conf. Ser., in press

Georganopoulos, M., \& Marscher, A. P. 1998, ApJ, 506, L11

Ghisellini, G., Celotti, A., Fossati, G., Maraschi, L., \& Comastri, A. 1998, MNRAS, 301, 451

Ghisellini, G., et al. 1999, A\&A, 348, 63

Giommi, P., et al. 1998, A\&A, 333, L5

Giommi, P., et al. 1999, A\&A, 351, 59

Giommi, P., Padovani, P., \& Perlman, E. 2000, MNRAS, 317, 743

Guainazzi, M., Vacanti, G., Malizia, A., O’Flaherty, K. S., Palazzi, E., \& Parmar, A. N. 1999, A\&A, 342, 124 
Hartman, R. C., et al. 2001, ApJ, 553, 683

Kataoka, J., et al. 1999, ApJ, 514, 138

Kataoka, J., et al. 2000, ApJ, 528, 243

Kirk, J. G., Rieger, F. M., \& Mastichiadis, A. 1998, A\&A, 333, 452

Krawczynski, H., Coppi, P. S., Maccarone, T., \& Aharonian, F. A. 2000, A\&A, 353, 97

Krawczynski, H., et al. 2001, ApJ, 559, 187

Lamer, G., \& Wagner, S. J. 1998, A\&A, 331, L13

Lawson, A. J., McHardy, I. M., \& Marscher, A. P. 1999, MNRAS, 306, 247

Malizia, A., et al. 2000, MNRAS, 312, 123

Mannheim, K. 1998, Science, 279, 684

Maraschi, L., et al. 1999, ApJ, 526, L81

Maraschi, L., Chiappetti, L., Fossati, G., Pian, E., \& Tavecchio, F. 2000, AdSpR, 25, 713

Maraschi, L., \& Tavecchio, F. 2001, Proc. of "X-ray Astronomy 2000" (Palermo, September 2000), Eds. R. Giacconi, L. Stella, and S. Serio, ASP Conf. Ser., in press (astro-ph/0107566)

Maraschi, L. 2001, in Relativistic Astrophysics: 20th Texas Symposium, AIP Conf. Proc. 586, Eds. J. C. Wheeler and H. Martel, p. 409

McHardy, I. 1999, in BL Lac Phenomenon, ASP Conf. Ser., Vol. 159, Eds. L. O. Takalo and A. Sillanpää, p. 155

Padovani, P., \& Giommi, P. 1995, ApJ, 444, 567

Perlman, E. S., Madejski, G., Stocke, J. T., \& Rector, T. A. 1999, ApJ, 523, L11
Petry, D., et al. 2000, ApJ, 536, 742

Pian, E., et al. 1998, ApJ, 492, L17

Rachen, J. P. 2000, astro-ph/0010289

Ravasio, M., et al. 2002, A\&A, in press (astro-ph/0201307)

Sambruna, R. M., et al. 1997, ApJ, 474, 639

Sambruna, R. M. 1999, Frascati Workshop 1999, May 24-29, 1999,

Vulcano, Italy, MEMSAIt, (astro-ph/9912060)

Sambruna, R. M., et al. 2000, ApJ, 538, 127

Sembay, S., et al. 1993, ApJ, 404, 112

Tagliaferri, G., et al. 2000, A\&A, 354, 431

Tagliaferri, G., et al. 2001, A\&A, 368, 38

Takahashi, T., et al. 1996, ApJ, 470, L89

Takahashi, T., Madejski, G., \& Kubo, H. 1999, APh, 11, 177

Takahashi, T., et al. 2000, ApJ, 542, L105

Tanihata, C., et al. 2000, ApJ, 543, 124

Tavecchio, F., Maraschi, L., \& Ghisellini, G. 1998, ApJ, 509, 608

Tavecchio, F., et al. 2001, ApJ, 554, 725

Treves, A., et al. 1999, Astronomische Nachrichten 320, n. 4, p. 317

Ulrich, M.-H., Maraschi, L., \& Urry, C. M. 1997, ARA\&A, 35, 445

Urry, C. M., et al. 1993, ApJ, 411, 614

Urry, C. M., et al. 1997, ApJ, 486, 799

Urry, C. M. 1999, APh, 11, 159

Vestrand, W. T., \& Sreekumar, P. 1999, APh, 11, 197

Wehrle, A. E., et al. 1998, ApJ, 497, 178

Zhang, Y. H., et al. 1999, ApJ, 527, 719 